\title{
Manejo da dor oncológica pela equipe de enfermagem
}

\author{
Management of oncological pain by the nursing team \\ Tratamiento del dolor oncológica por el equipo de enfermería
}

Recebido: 25/08/2021 | Revisado: 01/09/2021 | Aceito: 05/09/2021 | Publicado: 06/09/2021

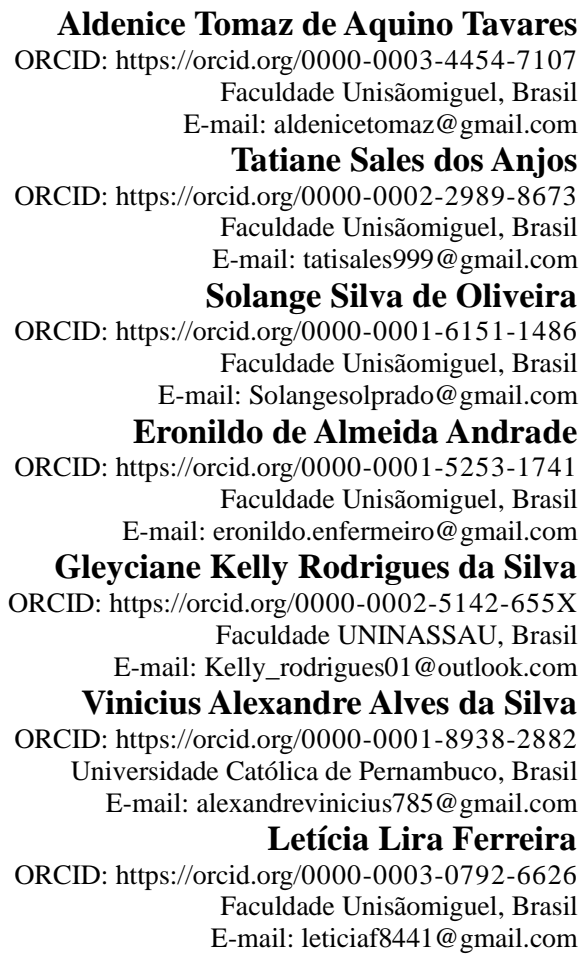

\section{Resumo}

O estudo aqui objetivou avaliar a dor em pacientes oncológicos e a forma que a equipe multidisciplinar vem cuidando desse sintoma tão difícil. O estudo é do tipo revisão da literatura. Os resultados apresentados foram em relação à dor oncológica, na presença de sinais e sintomas de difícil controle, o Enfermeiro tem um papel fundamental no alívio do desconforto e sofrimento dela, pois assiste o paciente durante as 24 horas e acompanha os aspectos e é uma das preocupações mais presentes no dia a dia do Enfermeiro que trabalha com esse tipo de paciente, principalmente, nas unidades de cuidados paliativos. Entende-se por cuidados paliativos todo cuidado ativo e global realizado a paciente cuja doença não respondeu ao tratamento curativo, entretanto, para poder proporcionar a estes cuidados, é fundamental que o profissional desenvolva conhecimentos holísticos sobre a dor e, por meio dele, alcance condições de avaliar e dimensionar a sua complexidade, compreende-se que a enfermagem é uma profissão insubstituível ao cuidar da sociedade, o quanto sua prática humana é ímpar na saúde do paciente. Nesse contexto, observa-se a necessidade de técnicas terapêuticas alternativas e complementares às convencionais para o manejo da dor, mas não dispensando tratamento com os fármacos de acordo com as orientações médicas para um resultado satisfatório.

Palavras-chave: Dor do câncer; Enfermagem oncológica; Estratégia de saúde.

\begin{abstract}
The aim of the present study was to evaluate pain in oncology patients and the way in which the multidisciplinary team has been taking care of this difficult symptom. The study is a literature review tipe. The results presented were in relation to cancer pain, in the presence of signs and symptoms of difficult control, the Nurse plays a fundamental role in relieving the discomfort and suffering of the same, because it assists the patient during the 24 hours and accompanies the aspects and is a of the concerns most present in the day-to-day of the nurse who works with this type of patient, mainly in the palliative care units. Palliative care is understood to mean all active and global care given to a patient whose illness has not responded to curative treatment; however, in order to provide such care, it is essential that the practitioner develops holistic knowledge about pain and, through it, achieves conditions to assess and size its complexity, it is understood that nursing is an irreplaceable profession when caring for society, how much its human practice is odd in the patient's health. In this context, the need for alternative and complementary therapeutic
\end{abstract}


techniques to conventional pain management is observed, but it does not require treatment with the drugs according to the medical guidelines for a satisfactory result.

Keywords: Cancer pain; Oncology nursing; Health strategies.

\section{Resumen}

El presente estudio tuvo como objetivo evaluar el dolor en pacientes con cancer y la forma en que el equipo multidisciplinario ha estado atendiendo este síntoma tan difícil. El estudio es un tipo de revision de la literatura. Los resultados presentados fueron en relación al dolor oncológico, ante la presencia de signos y síntomas de difícil control, la enfermera tiene un rol fundamental en el Alivio de su malestar y sufrimiento, ya que atiende a la paciente durante 24 horas y monitorea los aspectos y es una de las preocupaciones más presents en el día a día de los enfermeiros que trabajan con este tipo de pacientes, especialmente en las unidades de cuidado paliativos. Se entiende or cuidados paliativos todo cuidado active y global que se brinda a pacientes cuya enfermedad no ha respondido al tratamiento curativo, sin embargo, para brindar este cuidado es fundamental que los profesionales desarrollen un conocimiento holístico sobre el dolor y, a través de él, lograr condiciones para evaluar y escalar su complejidad, se entiende que la enfermería es una profesión insustituible en el cuidado de la sociedad, ya que su práctica humana es única en la salud del paciente. En este context, existe la necessidad de técnicas terápeuticas alternativas y complementarias a las convencionales para el manejo del dolor, pero no dispensando el Tratamiento con fármacos según las guías médicas para un resultado satisfactorio.

Palabras clave: Dolor en cáncer; Enfermería oncológica; Estrategias de salud.

\section{Introdução}

O Instituto Nacional do Câncer - INCA Brasil (2001) definiu câncer como sendo o crescimento anormal de células que invadem os tecidos e órgãos do corpo podendo espalhar-se para outras áreas e vem acompanhada de dor, e em 1979 a Organização Mundial de Saúde (OMS) definiu dor como uma experiência sensorial e emocional desagradável, associada a lesões reais ou potenciais.

A dor oncológica é uma “dor total”' tratando-se de uma síndrome, que vai além da lesão, abordando os fatores físicos, emocionais, espirituais e tem influência na expressão da queixa (Rigotti; Ferreira, 2005).

Em 1964, Cicely Saunders agregou ao entendimento da dor o conceito de "Dor Total" mostrando na dor pelo câncer que ela era constituída por dor física (e outros sintomas físicos de desconforto), dor emocional (ansiedade, de pressão), dor social (medo da separação, sensação de abandono, luto antecipatório) e dor espiritual, sendo posteriormente, acrescentadas as dores interpessoal, familiar e financeira.

O Instituto Nacional de Câncer-INCA (Brasil, 2001) relata que

[...] a dor moderada ou intensa está em um percentual referente a 30\% dos pacientes com câncer em tratamento, $60 \%$ a $90 \%$ com esta doença em situação avançada. No entanto, a dor oncológica pode ser aliviada de forma completa em cerca de $80 \%$ a $90 \%$ dos indivíduos.

O câncer é um conjunto de doenças que envolvem o crescimento celular anormal que pode invadir o local original e espalhar-se para outras partes do corpo com um crescimento desordenado (metástase) segundo informações do Instituto Nacional do Câncer (INCA).

Preocupados com a avaliação da dor, a Joint Comissionon Accreditation on Heathcare Organizations (JCA HO), a American Pain Society (APS) e a OMS propuseram a implementação sistemática de rotinas de avaliação, registro e controle da dor a serem incorporados pelos profissionais médicos e de enfermagem como a rotina de verificação de sinais vitais (pressão arterial, pulso, temperatura e respiração). Considerando-se, assim, a dor como o quinto Sinal Vital desde 2003,6. Por esse motivo é necessário aprimorar os conhecimentos nas condutas terapêuticas a fim de oferecer melhor condição de vida, considerando que ao vivenciar a dor oncológica o ser se torna frágeis, necessitando de uma atenção especial dos profissionais de saúde (Matos et al., 2015). 
Por terem vivenciado experiências ao cuidar de portadores de câncer, as autoras consideram de extrema importância abordar o tema, pois é significativo o avanço tecnológico do alívio da dor neste agravo.

Esse estudo foi desenvolvido para ajudar na construção do conhecimento a respeito do manejo da dor em pacientes portadores de câncer, considerando que, o enfermeiro tem papel primordial no processo de cuidar destas pessoas.

Esse trabalho vem com objetivo de investigar as medidas utilizadas pela equipe de enfermagem para o manejo da dor oncológica com ênfase de levar informações para equipe multidisciplinar, com o intuito de conhecer a importância da avaliação da dor e suas características em pacientes oncológicos, e assim poder traçar cuidados necessários e ações de melhoria no tratamento da dor oncológica.

\section{Metodologia}

O presente estudo foi elaborado a partir de uma revisão bibliográfica realizadas a partir de materiais distribuídos na literatura, com estudo exploratório, descritivo e qualitativo, assim como sites de referência sobre o assunto. O estudo foi desenvolvido a partir de artigos científicos disponíveis em duas bases de dados principais: (1) Scientific Electronic Library Online (Scielo); (2) (BVS); além de revistas da área de concentração específica (Enfermagem), como a Revista Brasileira de Enfermagem (REBEn); e em livros governamentais, em especial os publicados pelo INCA.

Todavia, este estudo busca uma reflexão histórica, cultural e política que irá interligar o processo saúde e doença com o cuidado do profissional de enfermagem. Fazendo assim uma análise da influência cultural antiga nos dias atuais.

Quanto aos critérios de inclusão, foram utilizados: artigos relacionados ao manejo da dor oncológica e condutas de Enfermagem do ano 2001 até 2015. Já como critério de exclusão, foi referido aos artigos que não tratasse da temática da dor oncológica, ou que relacionasse com a população pediatrica.

\section{Resultados}

Foram selecionados 8 artigos para esta revisão de literatura. O método das intervenções de enfermagem para controle da dor oncológica está apresentado na Tabela 1.

Tabela 1 - Caracterização dos artigos quanto ao tema, autoria, objetivo, local do estudo e resultados do estudo (n=17).

\begin{tabular}{|c|l|l|l|l|}
\hline Autores e Ano & \multicolumn{1}{|c|}{ Título } & Objetivo do estudo & Metodologia & \multicolumn{1}{c|}{ Resultado do estudo } \\
\hline $\begin{array}{c}\text { 1 Ferreira, R. G. } \\
\text { dos S., } \\
\text { Nascimento, J. L. } \\
2015\end{array}$ & $\begin{array}{l}\text { O manejo da dor } \\
\text { oncológica pelo } \\
\text { enfermeiro no cenário } \\
\text { da terapia intensiva em } \\
\text { hospital geral. }\end{array}$ & $\begin{array}{l}\text { Refere à compreensão e } \\
\text { planejamento assistencial } \\
\text { no que concerne o } \\
\text { cuidado ao paciente } \\
\text { oncológico. }\end{array}$ & $\begin{array}{l}\text { Revisão da } \\
\text { Literatura. }\end{array}$ & $\begin{array}{l}\text { Grau de conhecimento da dor que } \\
\text { influenciam a atuação dos } \\
\text { profissionais de enfermagem em } \\
\text { unidades oncológicas não é } \\
\text { assunto ainda Vislumbrado no } \\
\text { campo da apresentação científica, } \\
\text { uma lacuna de conhecimento. }\end{array}$ \\
\hline $\begin{array}{c}\text { 2 Borges, D. A.; } \\
\text { Oliveira, S. A. } \\
2015\end{array}$ & $\begin{array}{l}\text { Assistência de } \\
\text { enfermagem ao paciente } \\
\text { com dor oncológico }\end{array}$ & $\begin{array}{l}\text { Identificar as intervenções } \\
\text { de enfermagem para o } \\
\text { controle da dor } \\
\text { oncológica no paciente } \\
\text { com câncer. }\end{array}$ & $\begin{array}{l}\text { Revisão da } \\
\text { Literatura. }\end{array}$ & $\begin{array}{l}\text { A importância da capacitação } \\
\text { profissional para o controle da } \\
\text { dor; a avaliação da dor; e, a } \\
\text { intervenção de enfermagem para } \\
\text { controle da dor. }\end{array}$ \\
\hline $\begin{array}{c}\text { 3 Mariléia Stübe, } \\
\text { \& colaboradores } \\
2015\end{array}$ & $\begin{array}{l}\text { Percepçóes de } \\
\text { enfermeiros e manejo da } \\
\text { dor de pacientes } \\
\text { oncológicos }\end{array}$ & $\begin{array}{l}\text { Objetivo de qualificar a } \\
\text { assistência aos pacientes } \\
\text { oncológicos e contribuir } \\
\text { para a minimização da } \\
\text { dor. }\end{array}$ & $\begin{array}{l}\text { Revista Mineira } \\
\text { de Enfermagem } \\
\text { Pesquisa }\end{array}$ & $\begin{array}{l}\text { Os resultados sinalizam mudanças } \\
\text { que podem ser realizadas por } \\
\text { enfermeiros, diante da dor de } \\
\text { pacientes oncológicos e as ações } \\
\text { direcionadas para a redução da } \\
\text { mesma, descritas e analisadas } \\
\text { sequencialmente. }\end{array}$ \\
\hline
\end{tabular}




\begin{tabular}{|c|c|c|c|c|}
\hline $\begin{array}{c}4 \text { Carvalho, \& } \\
\text { colaboradores } 2015\end{array}$ & $\begin{array}{l}\text { Diagnósticos De } \\
\text { Enfermagem Para } \\
\text { Pacientes Com Dor } \\
\text { Oncológica Baseados Na } \\
\text { Cipe- }\end{array}$ & $\begin{array}{l}\text { Construir afirmativas de } \\
\text { diagnósticos de } \\
\text { enfermagem com base na } \\
\text { classificação internacional } \\
\text { para a prática de } \\
\left.\text { enfermagem (cipe }{ }^{\circledR}\right), \\
\text { versões } 1.0 \text { e } 1.1 \text {, para } \\
\text { pacientes com dor } \\
\text { oncológica. }\end{array}$ & Artigo original & $\begin{array}{l}\text { Com a literatura analisada e a } \\
\text { experiência clínica, a dor foi } \\
\text { classificada em dor oncológica de } \\
\text { origem física e de origem } \\
\text { psicogênica, nesta última, os } \\
\text { fatores psicológicos, sociais, } \\
\text { culturais e espirituais estão } \\
\text { presentes influenciando a } \\
\text { identificação e o tratamento da dor. }\end{array}$ \\
\hline 5 Cavalcanti, 2013 & $\begin{array}{l}\text { Evolução Da } \\
\text { Enfermagem: Um } \\
\text { Recorte Histórico, } \\
\text { Político E Cultural. }\end{array}$ & $\begin{array}{l}\text { Expor a trajetória } \\
\text { histórica e cultural da } \\
\text { enfermagem, Abordando } \\
\text { as políticas } \\
\text { governamentais no âmbito } \\
\text { da gestão nos cuidados à } \\
\text { saúde. }\end{array}$ & Artigo original & $\begin{array}{l}\text { O cuidado passou de uma } \\
\text { Assistência limitada ao círculo } \\
\text { familiar, que era transmitida de } \\
\text { uma geração a outra, para uma } \\
\text { Assistência técnico-científica, } \\
\text { através da formação de } \\
\text { profissionais em universidades, } \\
\text { Exercendo seus conhecimentos } \\
\text { em hospitais e UBS nos moldes } \\
\text { do SUS. }\end{array}$ \\
\hline 6 Schveitzer, 2012 & $\begin{array}{l}\text { Práticas Integrativas e } \\
\text { Complementares na } \\
\text { Atenção Primária em } \\
\text { Saúde: em busca da } \\
\text { humanização do } \\
\text { cuidado. }\end{array}$ & $\begin{array}{l}\text { Identificar como a } \\
\text { incorporação de práticas } \\
\text { integrativas e } \\
\text { complementares na } \\
\text { atenção primária } \\
\text { Em saúde tem auxiliado a } \\
\text { promover a humanização } \\
\text { do cuidado. }\end{array}$ & $\begin{array}{l}\text { Rev.de } \\
\text { Literatura }\end{array}$ & $\begin{array}{l}\text { Fomentar pesquisas sobre PIC, } \\
\text { inserir PIC nos cursos de } \\
\text { graduação e em treinamentos de } \\
\text { profissionais de saúde, promover } \\
\text { a colaboração internacional, } \\
\text { aproximar curadores tradicionais } \\
\text { e profissionais da APS e } \\
\text { organizar lista de fitoterápicos e } \\
\text { Plantas medicinais recomendadas } \\
\text { pelos sistemas de saúde. }\end{array}$ \\
\hline $\begin{array}{l}7 \text { Santini L. A. de } \\
2012\end{array}$ & $\begin{array}{l}\text { ABC do Câncer } \\
\text { Abordagens básicas para } \\
\text { o controle do Câncer. }\end{array}$ & $\begin{array}{l}\text { Políticas, ações } \\
\text { E programas } \\
\text { Pacto de gestão, pacto pela } \\
\text { vida e pacto em defesa do } \\
\text { SUS, que possui objetivos } \\
\text { e metas que devem ser } \\
\text { Repactuados anualmente. }\end{array}$ & INCA & $\begin{array}{l}\text { É a exigência de que, quando um } \\
\text { indivíduo busca o atendimento ou } \\
\text { quando surge um problema de } \\
\text { Impacto coletivo sobre a saúde, o } \\
\text { serviço correspondente esteja } \\
\text { capacitado para enfrentá-lo e } \\
\text { resolvê-lo até o nível da sua } \\
\text { competência. }\end{array}$ \\
\hline $\begin{array}{c}8 \text { Biasi, P. T. \& } \\
\text { colaboradores, } 2011\end{array}$ & $\begin{array}{l}\text { Manejo da dor no } \\
\text { paciente oncológico pela } \\
\text { equipe de enfermagem. }\end{array}$ & $\begin{array}{l}\text { Averiguar o desempenho e } \\
\text { assistência prestados pela } \\
\text { equipe de enfermagem, } \\
\text { através da aplicação da } \\
\text { escala de dor em pacientes } \\
\text { portadores de câncer frente } \\
\text { à prevenção e ao controle } \\
\text { da mesma, em uma } \\
\text { unidade de internação } \\
\text { oncológica. }\end{array}$ & Artigo original & $\begin{array}{l}\text { Os resultados indicaram que a } \\
\text { Equipe de enfermagem apresentou } \\
\text { algumas dificuldades dentro de } \\
\text { limitações na mensuração e } \\
\text { avalição da dor nos pacientes com } \\
\text { câncer, assim como no Manejo da } \\
\text { dor vivenciado pelo paciente e a } \\
\text { adequada terapêutica. }\end{array}$ \\
\hline $\begin{array}{l}9 \text { Roberta } \\
\text { Waterkemper., } \\
\text { Kenya Schmidt, } \\
2010\end{array}$ & $\begin{array}{l}\text { CUIDADOS } \\
\text { PALIATIVOS: a } \\
\text { avaliação da dor na } \\
\text { percepção de enfermeiras }\end{array}$ & $\begin{array}{l}\text { Revelar as concepções e } \\
\text { contribuições de } \\
\text { enfermeiras sobre a } \\
\text { avaliação da dor em } \\
\text { pacientes com câncer em } \\
\text { Cuidados paliativos, } \\
\text { através de uma proposta de } \\
\text { educação no trabalho } \\
\text { fundamentada nos } \\
\text { pressupostos da educação } \\
\text { Problematizadora de Paulo } \\
\text { freire. }\end{array}$ & Artigo original & $\begin{array}{l}\text { Apontaram para três categorias: o } \\
\text { significado da dor, a forma de } \\
\text { avaliação da dor praticada pelas } \\
\text { enfermeiras e as contribuições para } \\
\text { o cuidado. }\end{array}$ \\
\hline
\end{tabular}




\begin{tabular}{|c|c|c|c|c|}
\hline $\begin{array}{c}10 \text { Priscilla } \\
\text { Valladares Broca., } \\
\text { Márcia D.E., } \\
\text { Assunção, F. } 2010\end{array}$ & $\begin{array}{l}\text { Equipe de enfermagem } \\
\text { e comunicação: } \\
\text { contribuições para o } \\
\text { cuidado de enfermagem }\end{array}$ & $\begin{array}{l}\text { É identificar os elementos } \\
\text { que compõem o processo } \\
\text { de comunicação da } \\
\text { equipe de enfermagem, } \\
\text { analisar as estratégias de } \\
\text { comunicação utilizadas } \\
\text { pela equipe e discutir } \\
\text { suas influências no } \\
\text { cuidado de enfermagem. }\end{array}$ & Pesquisa & $\begin{array}{l}\text { A interação tem um grande papel } \\
\text { na equipe de enfermagem de } \\
\text { modo a aumentar a nossa } \\
\text { capacidade de influenciar e ser } \\
\text { influenciados quando se participa } \\
\text { de um processo de comunicação e, } \\
\text { dessa forma, favorecer o } \\
\text { desenvolvimento de um cuidado } \\
\text { efetivo para com o cliente. }\end{array}$ \\
\hline $\begin{array}{l}11 \text { Barbosa, J.A.A., } \\
\text { \& colaboradores, } \\
2008\end{array}$ & $\begin{array}{l}\text { Farmacoterapia } \\
\text { Adjuvante No } \\
\text { Tratamento } \\
\text { Da Dor Oncológica }\end{array}$ & $\begin{array}{l}\text { Avaliar o tratamento } \\
\text { farmacoterapêutico } \\
\text { adjuvante analgésico em } \\
\text { pacientes } \\
\text { Oncológicos. }\end{array}$ & Artigo original & $\begin{array}{l}\text { Os achados demonstraram } \\
\text { frequência em pacientes abaixo de } \\
60 \text { anos de idade, além de adoção } \\
\text { de uma politerapia e doenças } \\
\text { concomitantes que corroboram } \\
\text { como riscos para o tratamento. }\end{array}$ \\
\hline $\begin{array}{l}12 \text { Klaumann, P. R., } \\
\text { Wouk, A. F. P. F., } \\
\text { Sillas, T. } 2008\end{array}$ & Patofisiologia da dor. & $\begin{array}{l}\text { Abordar } \\
\text { A fisiopatologia do } \\
\text { processo doloroso, bem } \\
\text { como suas aplicações na } \\
\text { terapêutica analgésica. }\end{array}$ & $\begin{array}{l}\text { Revisão da } \\
\text { Literatura. }\end{array}$ & $\begin{array}{l}\text { Deve-se ter em mente que não } \\
\text { existem efeitos negativos da } \\
\text { utilização dos analgésicos, mas sim, } \\
\text { relacionados à escolha inadequada } \\
\text { ou a dose. }\end{array}$ \\
\hline $\begin{array}{l}13 \text { Andreya C. P. \& } \\
\text { colaboradores, } 2008\end{array}$ & $\begin{array}{l}\text { Dor Oncológica: os } \\
\text { cuidados de enfermagem }\end{array}$ & $\begin{array}{l}\text { O que torna premente o } \\
\text { desenvolvimento de novos } \\
\text { tratamentos para controle } \\
\text { da dor oncológica e o } \\
\text { treinamento dos } \\
\text { enfermeiros para o cuidado } \\
\text { do paciente de câncer com } \\
\text { dor. }\end{array}$ & $\begin{array}{l}\text { Artigo } \\
\text { Original }\end{array}$ & $\begin{array}{l}\text { Educar os profissionais que atuam } \\
\text { na oncologia para o esclarecimento } \\
\text { do público quanto ao medo } \\
\text { exagerado da dor neoplásica é de } \\
\text { extrema importância, para que os } \\
\text { pacientes que necessitarem de } \\
\text { terapias anti álgicas possam ser } \\
\text { assistidos de maneira mais } \\
\text { tranquila, sem medos ou fantasmas } \\
\text { que aumentam a ansiedade e } \\
\text { induzem a sofrimentos psicológicos } \\
\text { desnecessários que dificultando o } \\
\text { seu tratamento. }\end{array}$ \\
\hline $\begin{array}{l}14 \text { Célia, S.U.C. } \\
2008\end{array}$ & $\begin{array}{l}\text { A Necessária Atenção à } \\
\text { Família do Paciente } \\
\text { Oncológico }\end{array}$ & $\begin{array}{l}\text { Trazer uma reflexão sobre } \\
\text { as repercussões de uma } \\
\text { doença como o câncer para } \\
\text { as famílias dos enfermos. }\end{array}$ & $\begin{array}{l}\text { Revisão de } \\
\text { Literatura }\end{array}$ & $\begin{array}{l}\text { Pacientes e familiares devem ser } \\
\text { reconhecidos, pelos profissionais de } \\
\text { saúde, como atores sociais ativos, } \\
\text { participantes, corresponsáveis no } \\
\text { processo de tratamento, tendo } \\
\text { respeitadas as suas dificuldades } \\
\text { emocionais, sociais, econômicas e } \\
\text { culturais que impõem limites para o } \\
\text { enfrentamento à situação do câncer. }\end{array}$ \\
\hline $\begin{array}{l}15 \text { Ferreira, M. A. } \\
2006\end{array}$ & $\begin{array}{l}\text { A comunicação no } \\
\text { cuidado: uma questão } \\
\text { fundamental na } \\
\text { enfermagem }\end{array}$ & $\begin{array}{l}\text { Tem como objetivo } \\
\text { caracterizar, analisar } \\
\text { aspectos inerentes e } \\
\text { discutir a comunicação. }\end{array}$ & Pesquisa & $\begin{array}{l}\text { Nos resultados da pesquisa ora } \\
\text { apresentada, o dado importante que } \\
\text { marcou positivamente o cuidado } \\
\text { foi, justamente, as conversas que os } \\
\text { enfermeiros, auxiliares e técnicos } \\
\text { estabeleciam com os clientes no } \\
\text { decorrer do dia. Estas conversas } \\
\text { integravam o cliente ao meio social, } \\
\text { favorecia a interação dos clientes } \\
\text { entre eles próprios e com a família. }\end{array}$ \\
\hline $\begin{array}{c}\text { 16 Luís, F.R.S. } \\
2006\end{array}$ & $\begin{array}{l}\text { Política Nacional de } \\
\text { Práticas Integrativas e } \\
\text { Complementares no SUS }\end{array}$ & $\begin{array}{l}\text { Incorporar e programar a } \\
\text { PNPIC no SUS, contribuir } \\
\text { para o aumento da } \\
\text { resolubilidade do sistema e } \\
\text { ampliação do acesso à } \\
\text { PNPIC, promover a } \\
\text { racionalização das ações de } \\
\text { saúde, estimular as ações } \\
\text { referentes ao }\end{array}$ & $\begin{array}{l}\text { Ministério da } \\
\text { Saúde }\end{array}$ & $\begin{array}{l}\text { A melhoria dos serviços e o } \\
\text { incremento de diferentes } \\
\text { abordagens Configuram, assim, } \\
\text { prioridade do Ministério da Saúde, } \\
\text { tornando disponíveis opções } \\
\text { preventivas e terapêuticas aos } \\
\text { usuários do SUS. Esta Política } \\
\text { Nacional busca, portanto, } \\
\text { concretizar tal prioridade, }\end{array}$ \\
\hline
\end{tabular}




\begin{tabular}{|c|l|l|l|l|}
\hline & & $\begin{array}{l}\text { controle/participação } \\
\text { Social. }\end{array}$ & $\begin{array}{l}\text { imprimindo-lhe a necessária } \\
\text { segurança, eficácia e qualidade na } \\
\text { perspectiva da integralidade da } \\
\text { atenção à saúde no Brasil. }\end{array}$ \\
\hline $\begin{array}{c}17 \text { Miceli, V.P. } \\
2001\end{array}$ & $\begin{array}{l}\text { Dor crônica e } \\
\text { subjetividade em } \\
\text { oncologia }\end{array}$ & $\begin{array}{l}\text { O que requer uma } \\
\text { abordagem de tratamento } \\
\text { especializada e } \\
\text { multidisciplinar, de modo a } \\
\text { identificar e resolver os } \\
\text { problemas de comunicação } \\
\text { e mensuração da dor e a } \\
\text { ampliar os cuidados } \\
\text { terapêuticos oferecidos ao } \\
\text { paciente e suporte prestado } \\
\text { à família e cuidadores, e à } \\
\text { equipe de saúde. }\end{array}$ & $\begin{array}{l}\text { Artigo } \\
\text { Original }\end{array}$ & $\begin{array}{l}\text { A doença e a dor já não são mais, } \\
\text { isoladamente, o alvo do tratamento. } \\
\text { Deve-se entrelaçar diversos saberes } \\
\text { ampliando o foco de atenção e } \\
\text { cuidados para além do paciente, a } \\
\text { família que dele cuida e a equipe } \\
\text { que dele trata. }\end{array}$ \\
\hline
\end{tabular}

Fonte: Autores (2021).

Resultados encontrados foram que os artigos citados acima 1,2,3,4,9,10,13, objetivou descrever, identificar e qualificar as assistências de enfermagem nos cuidados com a dor oncológica, o 5 vem trazendo a história da enfermagem, lembrando que já na época da enfermeira Florence Nightingale preconizou que a enfermagem era uma arte e que precisa de treinamento técnico-científico, o 6 e 16 referem-se às práticas integrativas complementares de saúde. $\mathrm{O}$ artigo 7 já vem trazendo as políticas para a saúde.

O artigo 8,10,11 e 12 ainda cita o uso dos fármacos sem ou com acompanhamentos alternativos. Os artigos 14,15 e 17 vêm abordando a família, a comunicação como ajuda no tratamento dos pacientes de forma holística, mas a pesquisa para o manejo da dor oncológica com recursos alternativos ainda encontra-se ausente na maioria do tratamento do câncer, porém os autores citados trás na maioria a mesma linha de pensamento em relação ao conteúdo proposto na pesquisa, com a diferença de acrescentar alguns sintomas diferentes como: fadiga, mal estar, fraqueza, entre outros, mas finaliza com um sintoma mais temido pelo paciente e seus familiares que é a dor, o controle e o alívio da dor na assistência ao paciente oncológico tem sido imprescindível para a equipe multidisciplinar, na busca díspar de interferências quando se pode minimizar ou evitar problemas que acarretem transtornos físicos, emocionais e psicossociais aos portadores, assim como aos familiares.

\section{Discussão}

De acordo com a tabela acima foram encontrados mais artigos falando sobre a falta de qualificação dos profissionais de saúde no manejo da dor oncológica, mas também foram observados o início da enfermagem já com o uso das práticas saudáveis, que no momento atual já foi implementado na política da saúde a prática integrativa complementares de saúde e mesmo assim a enfermagem não faz uso regular das mesmas. Tem muitos artigos falando sobre o uso dos fármacos como o único meio de aliviar ou cessar a dor, mas também tem uns que vem abordando de forma inteligente com a parceria das práticas integrativas complementares de saúde, mas infelizmente são poucos. Outros artigos conseguiram trazer a família como ajuda nesse tratamento tão difícil e complicado para o paciente oncológico através da comunicação, religião de acordo com cada doutrina. Mas, a presente revisão é importante no sentido de fornecer o conhecimento da enfermagem, quanto a contribuição com os pacientes e aos seus familiares, seja de forma direta ou indiretamente, além, da consciência de que a saúde precisa de ajuda com um todo da equipe multidisciplinar para o cuidado com paciente oncológico principalmente na dor.

O papel da enfermagem é indispensável para um tratamento completo e humanizado e também incluindo os tratamentos paliativos que requer muitos dos nossos olhares holísticos na hora de se puncionar com os pacientes e os seus familiares como vem citado em um artigo na tabela acima. Neste sentido, nas últimas décadas o conhecimento, o conceito e as 
intervenções terapêuticas para a dor crônica do paciente oncológico tiveram uma grande evolução, porém, a capacitação do enfermeiro e equipe ainda é inadequada, ao enfermeiro cabe aperfeiçoar este cuidado, sendo um bom avaliador dos sintomas e suas intensidades, atuando preventivamente quanto às complicações indesejáveis, realizando um manejo adequado de lesões e limitações impostas pelo agravo da doença oncológica avançada.

\section{Considerações}

Durante nossa pesquisa foi possível identificar através de artigos, revistas e livros sobre oncologia que a grande maioria dos pacientes com câncer sofre de dor aguda ou crônica e que essa dor, muitas vezes, é subtratada, não apenas pelos enfermeiros, mas também por outros profissionais de saúde.

Foram observadas algumas irregularidades nos diagnósticos e registro da queixa de dor em relação a esses pacientes, levando-os, assim, a questionar um quadro de dor persistente motivando impacto na qualidade de vida e mudanças em suas atividades diárias.

Sabe-se que a dor não deve ser tratada apenas com fármacos, por que já existe a "Estratégia da OMS sobre Medicina Tradicional 2002-2005" (PNPIC). O conhecimento acerca dos aspectos emocionais e sociais que permeiam o problema é necessário implantar as experiências pioneiras na assistência terapêutica e, ainda, a necessidade de especialização do Enfermeiro direta ou indiretamente na qualidade da prestação do cuidado ao paciente.

Possibilitando aprimorar ou elaborar o cuidado de Enfermagem, focar o respeito nas conversas, pois mediante essas conversas possibilita um aconchego entre as pessoas, assim a união entre todos de forma holística.

O profissionalismo correto e seguro com a equipe multidisciplinar favorece o trabalho em equipe de forma cooperativa contribuindo para um atendimento humanizado e completo, assim coopera com a reabilitação com a saúde do paciente e do trabalhador. Ao assistir um paciente com dor é essencial respeitar duas ações que são indispensáveis para o diagnóstico.

Primeiro: o Enfermeiro tem que aceitar a queixa do paciente. Na ausência de escala de dor, o diagnóstico é realizado de acordo com as informações físicas, mentais ou emocionais da dor.

Segundo: é no olhar na mudança de aspectos não verbais que mostra a dor. Idem relevante: a avaliação da enfermagem é considerada a partir da: Qualidade da dor: aguda ou crônica; Comportamento do paciente; reconhecer motivos que colaboram com a dor e retorno do paciente a ela.

Contudo, se faz necessária a realização de mais estudos, correlacionando efeitos de manejos diferentes e seus respectivos benefícios ou não, assim como, a importância do profissional de enfermagem em lidar com as diversas situações e contextos.

\section{Referências}

Agarwal, R., \& Epstein, A. S. (2018). Advance care planning and End-of-LIFE decision making for patients with cancer. Rev. Semi. Enferm. Oncológica, 34(3), 316-326. https://doi.org/10.1016/j.soncn.2018.06.012.

Borges, D. A., \& Oliveira, S. A. (2015). Assistência de enfermagem ao paciente com dor oncológico. Trabalho de conclusão de curso de Pós-Graduação (TCC) em Oncológica Clínica. Universidade Católica de Goiás.

Biasi, P. T. (2016). Manejo da dor no paciente oncológico pela equipe de enfermagem. Rev. Perspectiva Erechim, 35(129), 157-66. http://www.u ricer.edu .br/site/pdfs/perspectiva/129_163.pdf.

Barbosa J. A. A., Belém, L. F., Sette, I. M. F., Carmo, E. S., Pereira, G. J. S., \& Júnior E. D. (2008). Farmacoterapia Adjuvante No Tratamento Da Dor Oncológica. Rev. Brasil. Em promoção da saúde, 21(2), 112-120. https://periodicos.unifor.br/RBPS/article/view/669/2214.

Célia, S. U. C. (2018). A Necessária Atenção à Família do Paciente Oncológico INCA. www.inca.gov.br/rbc/n_54/v01/pdf/revisao_7_pag_97a102.pdf

Andreya C. P., Tulli, C. S. C., \& Sinara Z. T. (2008). Dor Oncológica: os cuidados de enfermagem. blogspot.com/2008/07/artigo-dor-oncolgica-os-cuidadosde.html. 
Research, Society and Development, v. 10, n. 11, e472101119854, 2021

(CC BY 4.0) | ISSN 2525-3409 | DOI: http://dx.doi.org/10.33448/rsd-v10i11.19854

Ferreira, M. A. A. (2006). Comunicação no cuidado: uma questão fundamental na enfermagem. Rev Bras Enferm, 59(3), 327-30, https://www.sciel o.br/j/reben/a/qbBCcFvnLpKQkDzfjxGcpxs/abstract/?lang=pt.

Ferreira, R. G. S., Nascimento, J. L. (2016). O manejo da dor oncológica pelo enfermeiro no cenário da terapia intensiva em hospital geral. Rev. Saúde e Desenvolvimento, 8(4), 75-89. https://www.revistasuninter.com/revistasaude/index.php/saudeDesenvolvimento/article/view/408.

José, Ernesto Belizário. (2002). O próximo desafio reverter o câncer. Rev. Ciência Hoje, 31(184), 51-57, https://repositorio.usp.br/item/001253061.

Luís, F. R. S. (2017). Política Nacional de Práticas Integrativas e Complementares no SUS. bvsms.saude.gov.br/bvs/publicacoes/pnpic.pdf.

Miceli, V. P. (2001). Dor crônica e subjetividade em oncologia. www.inca.gov.br/rbc/n_48/v03/pdf/artigo5.pdf.

Stübe, M,. Cibele, T. C., Benetti, Eliane, R. R., Joseila, S.G., Stumm, E. M. F. (2015). Percepções de enfermeiros e manejo da dor de pacientes oncológicos. Rev. REME, 19(3), 704-710, www.reme.org.br/artigo/detalhes/1033-

Schveitzer, M. C., Esper, M. V. Silva, M. J. P. (2012). Práticas Integrativas e Complementares na Atenção Primária em Saúde: em busca da humanização do cuidado. Rev. Mundo da Saúde, 6(3), 442-451. https://bvsms.saude.gov.br/bvs/artigos/mundo_saude/praticas_integrativas_complemen tares_atencao_primaria.pdf

Marisaulina W. A. C., Anderley, A. C., Maria, M. N., \& Ana, C. R. C. (2015). Diagnósticos De Enfermagem Para Pacientes Com Dor Oncológica Baseados Na Cipe. Rev enferm UFPE, 9(1), 253-260. https://periodicos.ufpe.br/revistas/revistaenfermagem/article/download/10334/11033-

Cavalcanti, M. C. S. Evolução Da Enfermagem: Um Recorte Histórico, Político E Cultural. Trabalho de conclusão de curso (TCC) em enfermagem.

Pereira, R. D. M., Silva, W. W. O., Ramos, J. C. (2012). Revisão Integrativa Sobre Medidas Não Farmacológicas À Dor Oncológica. Rev. O Mundo da Saúde, 36(3): 442-451. https://periodicos.ufpe.br/revistas/revistaenfermagem/article/view/10391.

Broca, P. V., \& Ferreira, M. A. (2012). Equipe de enfermagem e comunicação: contribuições para o cuidado de enfermagem. Rev. Bras. Enferm, 65(1), 97103, https://www.scielo.br/j/reben/a/rxxwHhHCkZbGpD9M47DjDxp/?format=pdf\&lang=pt.

Roberta, W \& Kenya, S. R. (2010). Cuidados paliativos: a avaliação da dor na percepção de enfermeiras. Rev. Gaúcha de Enferm, 31(1), 84-91, https://www. sci elo.br/j/rgenf/a/XZdy3PYYKJmqYjwmGYMR7Zf/?lang=pt

Santini L. A. (2020). ABC do Câncer Abordagens básicas para o controle do Câncer. https://www.inca.gov.br/publicacoes/livros/abc-do-cancer-abordagensbasicas-para-o-controle-do-cancer

Klaumann, P. R., Wouk, A. F. P. F., Sillas, T. (2008). Patofisiologia da dor. Rev. Archives of Veterinary Science, 13(1), 1-12. revistas.ufpr.br/ve teri nary/article/download/11532/8022. 